\title{
A Study on Surveillance Service for Vehicles based on Cryptography using Attributes
}

\author{
Ho-Kyung Yang ${ }^{1}$, Hyun-Jong Cha ${ }^{2}$, and You-Jin Song ${ }^{3 *}$ \\ ${ }^{1}$ Professor, Division of Information Technology, Sunmoon University, Asan-si, Korea \\ ${ }^{2}$ Professor, Department of Multimedia, Chungwoon University, Incheon-si, Korea \\ 3*Professor, Department of Management, Dongguk University, Gyeongju-si, Korea \\ 1porori0421@naver.com, ${ }^{2}$ chj826@chungwoon.ac.kr, ${ }^{3 *}$ song@dongguk.ac.kr
}

\begin{abstract}
Massive amounts of data are sent and received in a vehicle traffic network, data involving countless vehicles, movements of people in the hundreds of millions, and their data, which get bundled with external environment data. The gathered data must at least be shared with legitimate users and depending on the situation data access privileges also need to be controlled. Because data between vehicles and the fog server may be subject to personal privacy infringement, a security technique must be introduced, such as encryption. To ensure secure sharing and use of data by users of a service, an encryption scheme is needed. Furthermore, delegation and revocation of decryption privileges are needed with this encryption scheme, concerning data encrypted based on user attributes. In this paper, we proposed a system that is safe from open recruitment attacks using an attribute-based authority delegation management method. The proposed system proved safe against publicly recruited attacks by malicious users using a semantic safety model. The proposed system can be applied to protect against smart car attacks. In the future, the encryption and calculation load will be reduced.
\end{abstract}

Keywords: Smart traffic network environment, Traffic surveillance service, Delegation, Revocation, Attribute

\section{Introduction}

A new customizable security service is needed for a variety of different network environments. The IoT infrastructure and the cloud environment for platform and service are being combined, and accordingly, in a future urban system, there would be increasing demands for technology that detects risks concerning each moving vehicle and which prevents accidents.

Massive amounts of data are sent and received in a traffic network, data on countless vehicles, movements of people in the hundreds of millions, and their data, which are bundled with external environment data. The existing mobile communication system has a structure in which data are gathered at a central server and processed. With such a structure, there would be a lack of time for making sophisticated judgments due to network latency and delays. Also, real-time processing is needed at the level of each of the moving vehicles and vehicles that are geographically close or have been in a similar situation in the past need to exchange information

Article history:

Received (April 9, 2021), Review Result (May 13, 2021), Accepted (July 5, 2021) 
to collect appropriate information such as accidents that had taken place nearby and identify information [1].

SDN designates a specific network structure and equipment for easy management of dispersed network devices with software. Using SDN, active and automated network resource management becomes possible for the telecommunication network operator, and as a result network management costs can be reduced and a prompt response can be made in an unexpected situation. While various implementations of network control have been proposed under the framework of SDN, the type of environment that gets applied to a network is limited. Escalation of privilege attacks is one of the security vulnerabilities with SDN. It arbitrarily grants privileges to an illegitimate user and it can cause a huge problem when it comes to access control [2].

Gathered data need to be at least shared by the user as appropriate to the purpose and depending on the situation data access privileges also need to be controlled. An encryption scheme is needed to require secure sharing and use of data for users of a service and to satisfy this requirement. Furthermore, the encryption scheme needs to have delegation and revocation capabilities, allowing decryption by legitimate users, with the data encrypted based on user attributes.

As a technical solution to this problem, this article describes how to provide vehicle traffic monitoring services using property delegation and termination techniques in a fog computing environment. The structure of this paper is as follows. Chapter 2 is related to research. In Chapter 3, this study explained the structure and procedure of secure encrypted communication so that falsification of smart cars and public offering attacks are impossible using attributebased authority delegation management methods. Finally, Chapter 5 conclusions are described.

\section{Related research}

\subsection{Smart traffic network environment model}

In the smart traffic network environment, C-ITS (Cooperative Intelligent Transport System) is operated as an open platform based on high-speed wireless communication between vehicles and infrastructure, and between vehicles and vehicles. It issues warnings to drivers and road users about getting into a car accident, thereby taking the level of traffic safety to another level [3].

ISO TC204 WG1 and WG18, working with CEN TC278 WG16, have concentrated their efforts on establishing a standard definition of C-ITS. So far, the definition is mostly centered on Europe. It aims for improving safety, system continuity, efficiency and convenience, and is defined as an overall part of ITS, providing risk information or recommendations via ITS communication with ITS-station information sharing and promoting responses [4].

To establish a computing infrastructure needed for the next-generation intelligent traffic system, a dedicated computing device called a processing node needs to be placed in the network. For example, a special router can not only forward packets but can also handle computing involving general application programs. IaaS cloud includes management such as the generation of computing resources to a certain extent in a network layer for a specific geographical area through fog computing [5]. Furthermore, a program interface similar to this which enables customizable computing instances also needs to be provided [6]. 


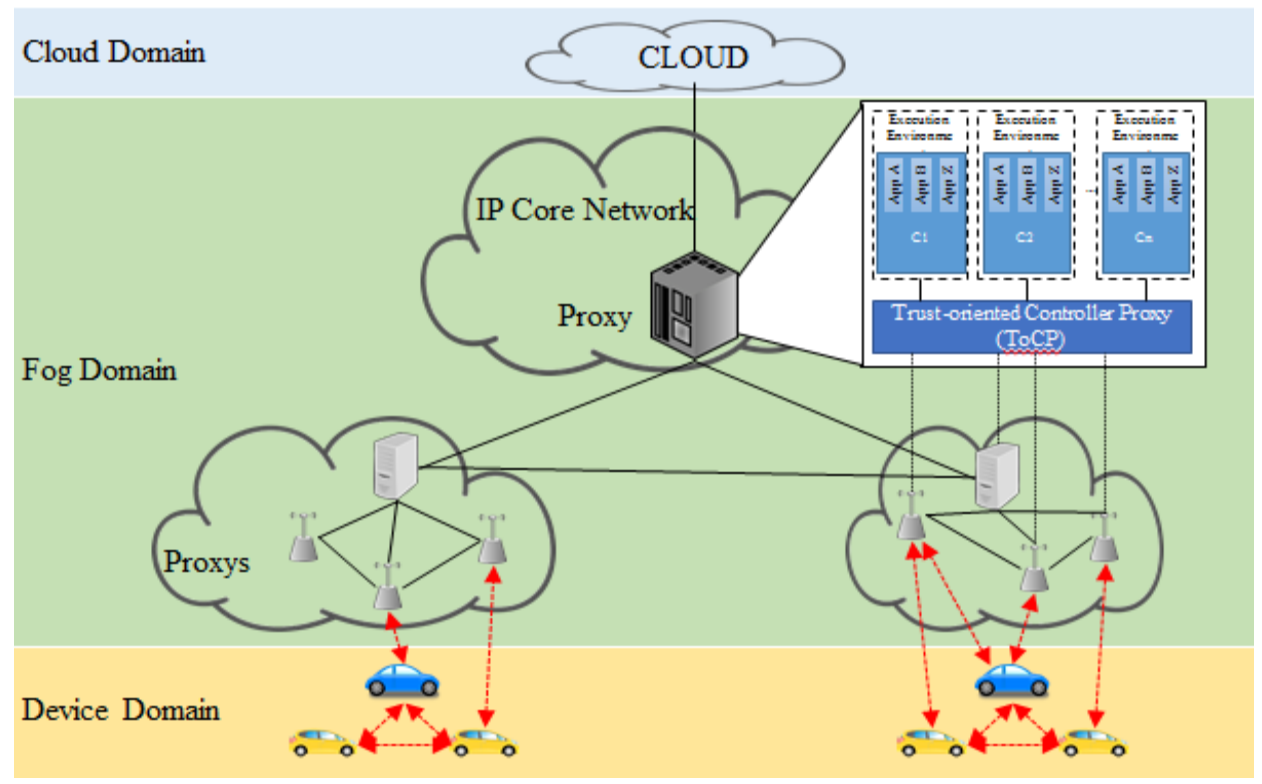

Figure 1. SDN-based VANET

\subsection{Fog computing}

Fog computing is a system that has been in the limelight due to the active introduction of IoT (Internet of Things) based on the concept proposed by Cisco. It is the Internet of things that has chips built into automobiles and various devices, including home appliances around us, to exchange various information via wired or wireless networks [4]. Sensors are attached to unmanned vehicles, eyeglasses, watches, shoes, and clothes to exchange physical information with servers for analysis and provision of information. Sensors are installed around the building to check the temperature and humidity, and through related equipment and devices, a comfortable environment may be maintained or used for security. By the way, there is a problem in storing all such data on a cloud server. There are physical things like storage capacity, but there are also time constraints to send and receive a lot of data [5]. That's why fog computing was introduced. In other words, the concept is that data that frequently appears is not stored in a remote cloud but is processed by nearby devices such as sensors and routers, and only the necessary data is sent to the cloud.

\subsection{Attribute-based encryption (CP-ABE) and attribute-based authority delegation possible Encryption (CP-ABTD)}

As we have made clear earlier, this paper proposes a new model that can use attribute-based encryption and attribute-based authority delegation techniques in ways to prevent threats such as smart car identity forgery and public recruitment attacks.

To that end, in attribute-based cryptography [7], the user's private key is associated with the attribute set, and the cryptography is associated with the attribute access structure. A cipher is decrypted if the user's set of private key attributes must be satisfied for a particular decryption policy within the cipher. Attribute-based cryptography does not describe the delegation of authority and delegation of authority.

Attribute-based withdrawal encryption [8] is an extended form of attribute-based proxy encryption that allows flexible attribute delegation and at the same time perform attribute 
withdrawal functions. This algorithm has three characteristics. First, an authority delegator with a private key associated with an attribute set can delegate his authority to the delegate. Second, the delegator can decide to re-delegate his authority to the delegator. Third, it is possible to withdraw the authority to withdraw the delegated authority.

\section{A service scenario with encryption that allows delegation and revocation of user attributes}

\subsection{System overview}

A system using an encryption scheme that allows delegation and revocation of user attributes encrypts data with an access structure based on user attributes and sends them to the fog server. A user that wants to share or use encrypted data of a service user requests the fog server for decryption privileges. The service user, after determining that the user that has requested decryption privileges is a legitimate user that should be able to access its data, generates a key for delegating attributes and sends it to the fog server. With this system, the fog server has to keep an attribute delegate list and attribute revocation list. The fog server checks the attribute delegate list and re-encrypts the data with a key that delegates the attribute and provides the data to the user.

\subsection{Attribute-based encryption}

Ciphertext-Policy Attribute-Based Threshold Decryption with Flexible Delegation and Revocation of User Attributes (CP-ABTD: ciphertext Policy Attribute-Based Threshold decryption whit flexible delegation and Revocation of User Attribute) is an extension of attribute-based encryption that allows flexible attribute delegation and revocation of user attributes. CP-ABTD has three different distinctive qualities. First, a delegator with a private key associated with an attribute group can delegate its privileges to the delegate. Second, the delegator can decide whether an entity can be delegated its privileges. Third, attribute revocation is possible.

CP-ABTD is made up of a total of seven algorithms, namely Setup, KeyGen, Encrypt, Delegate, m-Delegate, m-Decrypt, and Decrypt. The participants are the delegator, delegate, TA (Trusted Authority), and proxy.

Setup and KeyGen are performed by the TA; Encrypt and Delegate by the delegator; mDelegate and m-Decrypt by the proxy; and Decrypt by the delegate.

\subsection{Overview of the system operation}

[Figure 3] briefly shows the structure and operating procedure of the proposed system. The proposed system receives the data created by the smart card via the surrounding infrastructure. At this time, from the data to be transferred, confidential data such as personal information is encrypted to guarantee confidentiality. However, in a smart transportation network environment, the smart infrastructure has the constraint that it does not have sufficient computational and cancer decoding resources.

The proposed system consists of a delegator (vehicle), a delegate (police), a trusted authority (TA), and a policy administrator (Proxy). The proposed system was designed to allow the delegator to arbitrarily delegate and withdraw his or her authority under CP-ABTD. 


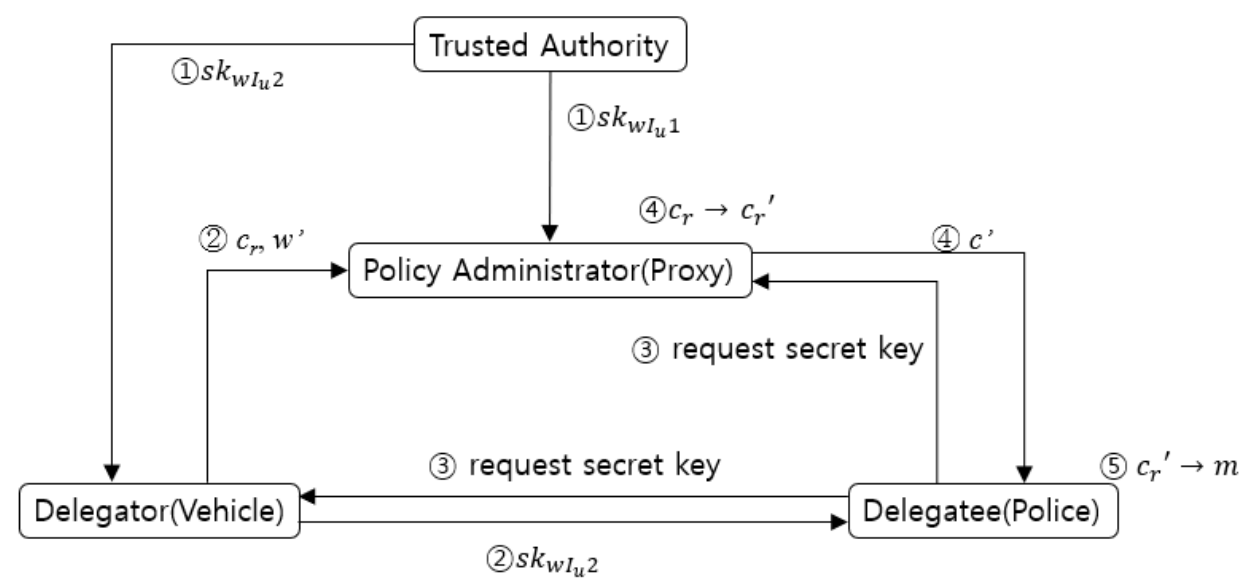

Figure 3. Proposed system configuration and operating procedure

The following describes the overview of a service in which vehicle traffic is monitored for traffic rule violations using an encryption scheme with attribute delegation and revocation capabilities.

First, Trusted Authority (TA) uses the $\operatorname{Setup}(k)$ algorithm to define system parameters and generates the public key $p k$ and the master key $m k$. Using the $\operatorname{KeyGen}\left(m k, w, I_{u}\right)$ algorithm, generate the two fog distribution keys $\boldsymbol{s} \boldsymbol{k}_{\boldsymbol{w} \boldsymbol{I}_{\boldsymbol{u}} \mathbf{1}}$ and $\boldsymbol{s} \boldsymbol{k}_{\boldsymbol{w} \boldsymbol{I}_{\boldsymbol{u}} \mathbf{2}}$ which are related to the vehicle attribute $\mathrm{W}$ and public key $I_{u}$. Then, distribute $\boldsymbol{s} \boldsymbol{k}_{\boldsymbol{w} \boldsymbol{I}_{\boldsymbol{u}} \mathbf{1}}$ to the fog server, and $\boldsymbol{s} \boldsymbol{k}_{\boldsymbol{w} \boldsymbol{I}_{\boldsymbol{u}} \mathbf{2}}$ to the vehicle.

Second, the vehicle sends the ciphertext $c_{r}$, which is data encrypted with the Encrypt ( $m, r$, $p k$ ) algorithm, to the database of the fog server. The data here generally refers to vehicle sensor data. The vehicle defines $w^{\prime}$ based on its attribute group $w$ to delegate decryption privileges for the ciphertext $c_{r}$ to the police vehicle. It generates a private key share $\boldsymbol{s} \boldsymbol{k}_{\boldsymbol{w} \boldsymbol{I}_{j} \mathbf{2}}$ for the police vehicle and the fog key $\boldsymbol{s} \boldsymbol{k}_{\boldsymbol{w} \rightarrow \boldsymbol{w}}$, that delegates attribute with its private key share $\boldsymbol{s}_{\boldsymbol{w} \boldsymbol{I}_{\boldsymbol{u}} \mathbf{2}}$ and the police vehicle $w$ 's public key $I_{j}$. It then sends each key respectively to the police vehicle $\left(\boldsymbol{w}^{\prime}, \boldsymbol{s k}_{\boldsymbol{w}^{\prime} \boldsymbol{I}_{\boldsymbol{i}} \boldsymbol{2}}\right)$ and the fog server $\left(\boldsymbol{s} \boldsymbol{k}_{\boldsymbol{w} \rightarrow \boldsymbol{w}^{\prime}}\right)$.

Third, to access the vehicle's data ID, the police vehicle requests the fog server for a decryption token (attribute group and ciphertext).

Fourth, the fog server generates a private key share $\boldsymbol{s} \boldsymbol{k}_{\boldsymbol{w} \boldsymbol{I}_{j} \mathbf{1}}$ for the police vehicle with its private key share $\boldsymbol{s} \boldsymbol{k}_{\boldsymbol{w} \boldsymbol{I}_{\boldsymbol{u}} \mathbf{1}}$, the fog key $\boldsymbol{s} \boldsymbol{k}_{\boldsymbol{w} \rightarrow \boldsymbol{w}}$, and the attribute group $w^{\prime}$ defined by the vehicle. The ciphertext $c_{r}$ is taken and re-encrypted as $c_{r}^{\prime}$ with the police vehicle's public key $\boldsymbol{I}_{\boldsymbol{j}}, \boldsymbol{s} \boldsymbol{k}_{\boldsymbol{w} / \boldsymbol{I}_{\boldsymbol{j}}} \mathbf{1}$. The re-encrypted ciphertext $c^{\prime}{ }_{r}$ is then sent to the police vehicle.

Finally, the police vehicle decrypts with the key $\boldsymbol{s}_{\boldsymbol{w}_{1} \boldsymbol{I}_{j} 2}$ and ciphertext $c^{\prime}$ received from the fog server and the vehicle and obtains an identifier data ID.

\section{Analysis}

Compared to existing public key-based cryptography and identity-based cryptography, attribute-based cryptography has the advantage of being more secure from malicious conspiracy attacks. However, it cannot handle user attribute delegation and withdrawal features when compared to attribute-based authority delegation encryption methods. 
Cryptographic algorithms that allow attribute-based delegation of authority can provide the ability to delegate or withdraw properties compared to existing attribute-based cryptographic algorithms. However, it does not present a realistically clear model. In addition, since it is not safe against the loss or tampering of the attribute conversion key, it has a weakness that a malicious external attacker can perform an attribute conversion key modulation attack.

Therefore, in this paper, we proposed a system that is safe against modulation attacks and collusion attacks based on the CP-ABTD authority delegation method. The proposal system can determine legitimate access through an authority auditor to withdraw authority delegation. Here, the meaning of the legitimacy judgment for the access of the P-delegator is that each authority examination given the share of the attribute conversion key divided into the dynamic threshold password grasps the identity of the delegator. The original attribute conversion key is generated by using the share of the threshold value that approves the access after the operation.

To view encrypted data-Additional dynamic threshold cryptography was applied to delegate the authority to view encrypted data to a delegate. As a result, it is possible to solve the problem of falsification that cannot be solved only by the technique of delegation of authority.

Table 1. Comparison of proposed systems

\begin{tabular}{|c|c|c|c|}
\hline & CP-ABE & CP-ABTD & Proposed systems \\
\hline Attribute withdrawal & $\times$ & $\bigcirc$ & $\bigcirc$ \\
\hline Delegation of authority & $\times$ & $\times$ & $\bigcirc$ \\
\hline Public offering attack & $\bigcirc$ & $\bigcirc$ & $\bigcirc$ \\
\hline Modulation attack & $\times$ & $\times$ & $\triangle$ \\
\hline
\end{tabular}

*Note: (O: Excellent, $\triangle$ : Normal, x: Insufficient)

\section{Conclusions}

In the age of the Internet, too many sensors and peripheral devices will be able to communicate with each other. In the future, based on artificial intelligence technology, it is expected to develop rapidly with autonomous vehicles. However, unlike what can have the convenience of a smart car, hacking the password of the smart car, tracking the location, or using it maliciously by disguising the identity of the owner of the smart car.

Too many security threats have been discovered. To solve these potential security threats, we discuss in this paper an attribute-based authority delegation cipher with a proxy (which performs the role of authority auditor) in an edge computing environment. We proposed a secure access control system for smart vehicles that use both computerization technology and threshold encryption technology.

The proposed system can transfer encrypted data in consideration of information leakage of smart vehicles. It consists of a trusted peer reviewer, a policy manager, and a certificate authority, and blood from the system-the delegator (police) re-encrypts and conveys information about a particular vehicle and then decrypts and drives it. I tried to be able to open and stop the car on behalf of someone (user, information constructor).

In this paper, attribute-based authority delegation techniques and threshold encryption techniques can be used to provide stability against malicious user recruitment attacks in an edge computing environment-based environment. However, it has the drawback of requiring a reencryption key to be generated each time an attribute withdrawal occurs, which is computationally expensive. In the future, I will try to find a way to reduce these loads. 


\section{Acknowledgments}

This research was supported by the Basic Science Research Program through the National Research Foundation of Korea (NRF) funded by the Ministry of Education (2019R1F1A1056507).

\section{References}

[1] S Chalkiadakis, Charis, P. Iordanopoulos, and E. Mitsakis, "Training opportunities for intelligent transport systems and cooperative intelligent transport systems," arXiv preprint arXiv:2010.12037 (2020)

[2] K. Tange, M. De Donno, X. Fafoutis, and N. Dragoni, "A systematic survey of industrial internet of things security: Requirements and fog computing opportunities," In IEEE Communications Surveys and Tutorials, vol.22, no.4, pp.2489-2520, (2020)

[3] H. J. Cha, H. K. Yang, J. M. Kim, and Y. J. Song, "Design of monitoring system of rule violation using ABPRE in the fog computing environment," Asia-pacific Journal of Applied Science and Engineering for Better Human Life 6, pp.29-31, (2016)

[4] J. S. Hurwitz and D. Kirsch, "Cloud computing for dummies," John Wiley and Sons, pp.56-74, (2020)

[5] Y. Xiao, M. Krunz, H. Volos, and T. Bando, "Driving in the fog: Latency measurement, modeling, and optimization of LTE-based fog computing for smart vehicles," In 2019 16th Annual IEEE International Conference on Sensing, Communication, and Networking (SECON), pp.1-9, June, (2019)

[6] J. R. Gudeme, S. K. Pasupuleti, and R. Kandukuri, "Attribute-based public integrity auditing for shared data with efficient user revocation in cloud storage," Journal of Ambient Intelligence and Humanized Computing, vol.12, no.2, pp.2019-2032, (2021)

[7] R. R. Al-Dahhan, Q. Shi, G. M. Lee, and K. Kifayat, "Survey on revocation in ciphertext-policy attribute-based encryption," Sensors, vol.19, no.7, p.1695, (2019)

[8] M. Ali, M. R. Sadeghi, and X. Liu, "Lightweight revocable hierarchical attribute-based encryption for the internet of things," IEEE Access, vol.8, pp.23951-23964, (2020) 


\section{Authors}

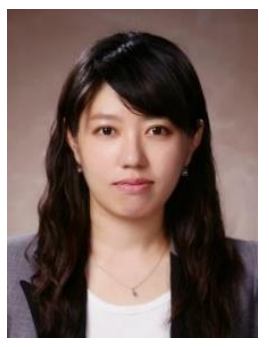

\section{Ho-Kyung Yang}

She received a B.S. and M.S in Computer science from Kwangwoon University, Seoul, Korea, in 2005 and 2007. She also received an M.S. and $\mathrm{Ph} . \mathrm{D}$. in Defense Acquisition from Kwangwoon University, Seoul, Korea, in 2010 and 2013. Her research interests include defense acquisition, network security, and network control architecture.

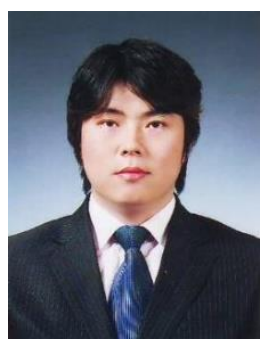

\section{Hyun-Jong Cha}

He received a B.S. and M.S in Computer science from Kwangwoon University, Seoul, Korea, in 2005 and 2008. He also received an M.S. and $\mathrm{Ph} . \mathrm{D}$. in Defense Acquisition from Kwangwoon University, Seoul, Korea, in 2011 and 2014. His research interests include defense acquisition, network security, and network control architecture.

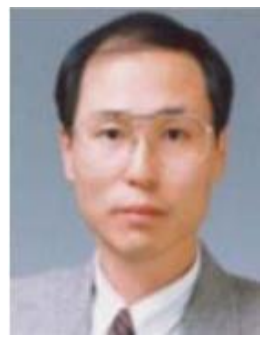

\section{You-Jin Song}

He received a Ph.D. in the Department of Information Security, Tokyo Institute of Technology University in Japan. He was work and research various security services and protocols at ETRI (Electronics and Telecommunications Research Institute) from 1988 and 1996 in Korea. He is a professor in the department of business and administration, Dongguk University Gyeongju Campus, Korea since 1996 and now. His research interest is Blockchain and IoT security services and Privacy-awareness and its applications. 